\title{
Long-Term Outcomes of NSAID-Induced Small Intestinal Injury Assessed by Capsule Endoscopy in Korea: A Nationwide Multicenter Retrospective Study
}

Ki-Nam Shim*, Eun Mi Song*, Yoon Tae Jeen ${ }^{\dagger}$, Jin-Oh Kim, Seong Ran Jeon ${ }^{\ddagger}$, Dong Kyung Chang ${ }^{\S}$, Hyun Joo Song"l, Yun Jeong Lim", Jin Soo Kim", Byong Duk Ye**, Cheol Hee Park ${ }^{\dagger+}$, Seong Woo Jeon ${ }^{\ddagger \neq}$, Jae Hee Cheon ${ }^{\$ \S}$, Kwang Jae Lee ${ }^{\|l\|}$, Ji Hyun Kim $^{\text {III }}$, Byung Ik Jang ${ }^{\# \#, ~ J e o n g ~ S e o p ~ M o o n * * *, ~ H o o n ~ J a e ~ C h u n ~}{ }^{\dagger}$, and Myung-Gyu Choi ${ }^{\#}$; The Korean Gut Image Study Group

*Department of Internal Medicine, Ewha Womans University School of Medicine, ${ }^{\dagger}$ Department of Internal Medicine, Korea University College of Medicine, ${ }^{\ddagger}$ Department of Internal Medicine, Soonchunhyang University College of Medicine, ${ }^{s}$ Department of Internal Medicine, Samsung Medical Center, Sungkyunkwan University School of Medicine, Seoul, "Department of Internal Medicine, Jeju National University College of Medicine, Jeju, "Department of Internal Medicine, Dongguk University College of Medicine, Goyang, "Department of Internal Medicine, The Catholic University of Korea College of Medicine, ${ }^{* *}$ Department of Gastroenterology, University of Ulsan College of Medicine, ${ }^{\text {t十 }}$ Department of Internal Medicine, Hallym University College of Medicine, Seoul, ${ }^{\ddagger \pm}$ Department of Internal Medicine, Kyungpook National University School of Medicine, Daegu, ${ }^{s s}$ Department of Internal Medicine, Yonsei University College of Medicine, Seoul, "II"Department of Gastroenterology, Ajou University School of Medicine, Suwon, "Department of Gastroenterology, Inje University College of Medicine, Busan, \#"Department of Internal Medicine, Yeungnam University College of Medicine, Daegu, and ${ }^{* * *}$ Department of Internal Medicine, Inje University College of Medicine, Seoul, Korea

See editorial on page 697.

Background/Aims: We evaluated the long-term outcome and clinical course of patients of nonsteroidal anti-inflammatory drug (NSAID)-induced small intestinal injury by performing capsule endoscopy (CE). Methods: A multicenter retrospective study was conducted using data collected from the $\mathrm{CE}$ nationwide database registry, which has been established since 2002. Results: A total of 140 patients ( 87 males; mean age, $60.6 \pm 14.8$ years) from the CE nationwide database registry $(n=2,885)$ were diagnosed with NSAID-induced small intestinal injury and enrolled in our study. Fortynine patients (35.0\%) presented with a history of aspirin use and an additional 49 (35.0\%) were taking NSAIDs without aspirin. The most prominent findings after performing $\mathrm{CE}$ were multiple ulcerations ( $n=82,58.6 \%)$ and erosions or aphthae $(n=32,22.9 \%)$. During the follow-up period (mean, 15.9 \pm 19.0 months; range, 0 to 106 months), NSAID-induced small intestinal injury only recurred in six patients (4.3\%). Older age and hypertension were positive predictive factors for recurrence. Conclusions: These results suggest that the recurrence of NSAID-induced small bowel injury was not frequent in the presence of conservative treatment. Therefore, the initial diagnosis using CE and the medication history are important. (Gut Liver 2015;9:727-733)
Key Words: Anti-inflammatory agents, non-steroidal; Capsule endoscopy; Outcome

\section{INTRODUCTION}

Nonsteroidal anti-inflammatory drugs (NSAIDs) are the most commonly prescribed drugs worldwide. Their popularity attests to their efficacy as anti-inflammatory, analgesic, and antipyretic agents. An estimated 30 million people worldwide take NSAIDs daily. ${ }^{1}$ However, NSAID use is limited by their associated gastrointestinal (GI) toxicity. These drugs can cause serious injury to any part of the GI tract, including life-threatening complications such as bleeding or perforation. ${ }^{2}$ The worldwide prevalence of NSAID-associated gastric and duodenal ulcers is 9\% to $22 \%$, with severe hemorrhage or perforation occurring in $<1 \%$ of patients annually.

The serious problem of NSAID-induced small intestinal injury has recently become a topic of great interest to gastroenterologists, as capsule endoscopy (CE) and double-balloon endoscopy are available for detecting small intestinal lesions. ${ }^{4-8}$ NSAIDinduced small intestinal injury had been under-examined or even ignored in clinical situations prior to the availability of CE. ${ }^{9}$ Several CE studies have been performed in NSAID users. Goldstein et al. ${ }^{10}$ reported small bowel mucosal breaks in 55\% of healthy volunteers who had taken naproxen for 2 weeks. Graham et al. ${ }^{11}$ performed CEs in patients with arthritis who had

\footnotetext{
Correspondence to: Yoon Tae Jeen

Department of Internal Medicine, Korea University College of Medicine, 73 Inchon-ro, Seongbuk-gu, Seoul 02841, Korea

Tel: +82-2-920-6555, Fax: +82-2-953-1943, E-mail: ytjeen@korea.ac.kr

Received on April 7, 2014. Revised on June 23, 2014. Accepted on June 27, 2014. Published online December 5, 2014

Ki-Nam Shim and Eun Mi Song contributed equally to this work as first authors.

pISSN 1976-2283 eISSN 2005-1212 http://dx.doi.org/10.5009/gnl14134

@) This is an Open Access article distributed under the terms of the Creative Commons Attribution Non-Commercial License (http://creativecommons.org/licenses/by-nc/4.0) which permits unrestricted non-commercial use, distribution, and reproduction in any medium, provided the original work is properly cited.
} 
been using NSAIDs for at least 3 months and reported the incidence of small intestinal mucosal injury to be as high as 71\%.

Previous studies have focused mainly on the incidence of NSAID-induced small intestinal injury in short-term and chronic NSAID users or on the protective effect of concurrent medication in patients with NSAID-induced small intestinal injury. However, data on the long-term outcome or natural course of NSAID-induced small intestinal injury are limited. Thus, we evaluated the long-term outcomes and clinical courses of patients diagnosed with NSAID-induced small intestinal injury based on the data from the Korean capsule endoscopy nationwide database registry.

\section{MATERIALS AND METHODS}

\section{Korean capsule endoscopy nationwide database registry}

CE has been performed at 23 tertiary hospitals in Korea since 2002 for 12 years, and the data have been registered in a common database. The database includes age, gender, indication for $\mathrm{CE}$, key CE findings, diagnosis post CE, bowel preparation method, and the corrective therapies applied to the patients.

\section{CE procedure}

Patients were examined using PilCam SB ${ }^{\circledR}$ (SB1/SB2) (Given Imaging, Yogneam, Israel) and MiroCam ${ }^{\circledR}$ (Intramedic, Seoul, Korea). Written informed consent was obtained from all patients before the procedure. CE was performed after a 12-hour fast and bowel preparation including ingestion of 2-L polyethylene glycol solution. The digital information recorded was downloaded onto a computer, and the images were analyzed using the proprietary RAPID software (Given Imaging).

\section{Patients and data collection}

Subjects who were diagnosed with NSAID-induced small intestinal injury were identified from the database between January 2002 and August 2012. The term "NSAID-induced small intestinal injury" included the following: (1) all small intestinal injuries observed by CE; (2) history of NSAID use prior to the initial CE; (3) suspected small bowel injuries based on performing upper and lower GI endoscopy; and (4) exclusion of other causes of local disease (malignant tumors, inflammatory bowel disease, infectious disease, and so forth). ${ }^{12}$

Among the 2,885 patients registered in the database, 140 with NSAID-induced small intestinal injury from 14 centers were enrolled in the study. In addition to registry data, other data were collected via chart review and telephone interviews. The following data were collected: (1) presence or absence of underlying disease in the patient; (2) mode of bleeding; (3) drug history such as type of drug and period of medication; (4) laboratory levels of hemoglobin and albumin; (5) follow-up period; (6) treatment method; (7) presence or absence of recurrence; and (8) any change in diagnosis during the follow-up period.

The definition of recurrence of NSAID-induced small intestinal injury was as follows: (1) history of diagnosis as NSAIDinduced small intestinal injury and recurring symptoms or anemia and findings of the same lesion as that found upon initial diagnosis via CE or (2) history of diagnosis as NSAID-induced small intestinal injury and recurring symptoms or anemia and suspected recurrence based on negative findings on other examinations such as upper and lower GI endoscopy except CE.

\section{Statistical analysis}

The results are presented as means \pm standard deviation or ranges for quantitative data and as frequencies (percentage)

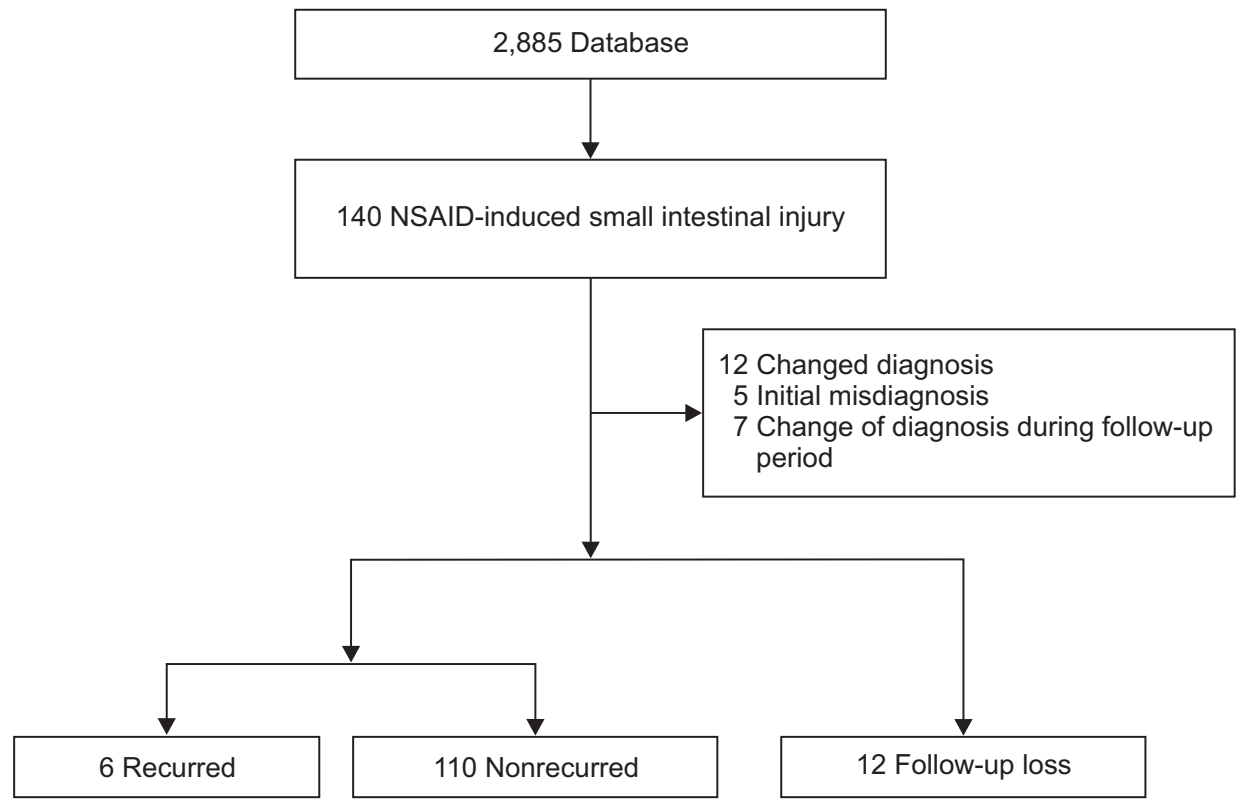

Fig. 1. Flow chart of the study subjects. NSAID, nonsteroidal anti-inflammatory drug. 

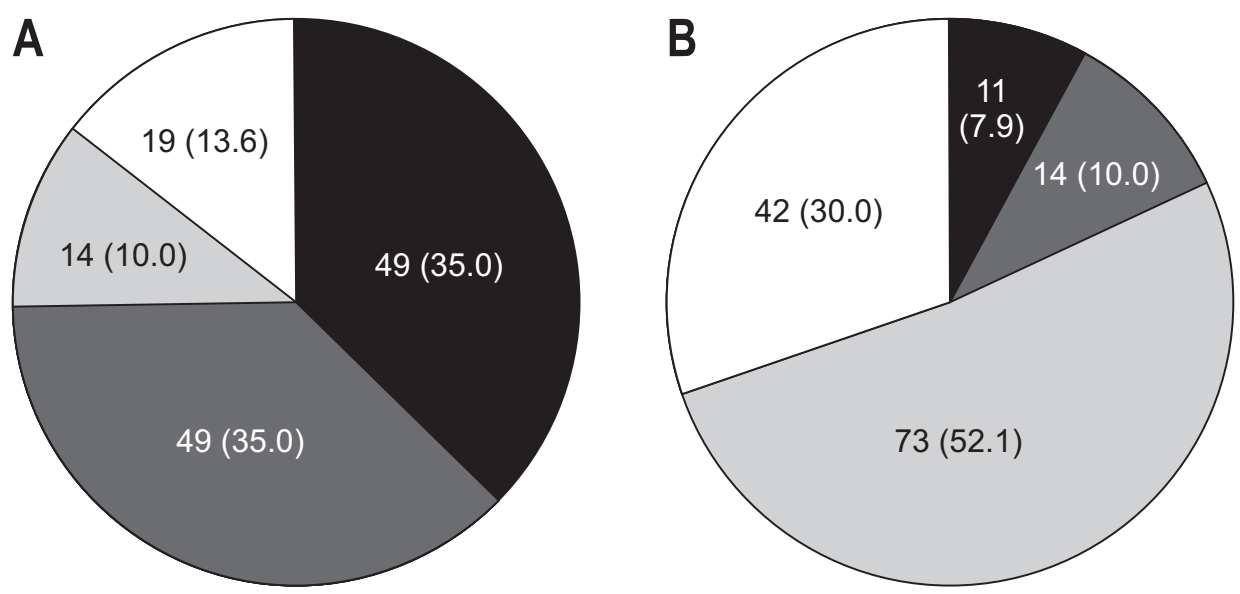

Less than $1 \mathrm{wk}$

1 wk-3 mo

More than 3 mo

Unknown
Fig. 2. Type of medication (A) and the period of nonsteroidal anti-inflammatory drug (NSAID) prescription (B). Data are presented as number (\%). for categorical data. Data were analyzed using Student t-test and the chi-square test. All statistical anlyses were performed using the SPSS version 16.0 for Windows software (SPSS Inc., Chicago, IL, USA). A p-value $<0.05$ was considered to indicate significance.

\section{RESULTS}

\section{Enrollment and characteristics of the patients}

Among the 2,885 patients registered in the database, 140 patients from 14 centers were diagnosed with NSAID-induced small intestinal injury. Twelve patients were lost to follow up. Twelve of 140 patients who were initially diagnosed experienced a change in diagnosis during the follow-up period. Therefore, 116 patients were included in the disease recurrence analysis. Finally, six patients were diagnosed with recurrence of NSAID-induced small intestinal injury (Fig. 1).

Aspirin ( $n=49,35.0 \%)$ and NSAIDs other than aspirin $(n=49$, $35.0 \%$ ) were the most frequently prescribed drugs in patients with NSAID-induced small intestinal injury. Ten percent $(n=14)$ of patients were taking both aspirin and other NSAIDs concurrently. More than half of patients were taking medications for more than 3 months ( $n=73,52.1 \%$ ) (Fig. 2).

The mean age of the patients was $60.6 \pm 14.8$ years (range, 23 to 90 years). The study group included 87 males (62.1\%) and 53 females (37.9\%). The most common underlying disease was hypertension (42.9\%). Diabetes (18.6\%), cardiovascular disease (21.4\%), and arthritis (19.2\%) were also common. The total

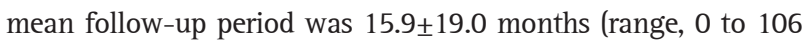
months). Anemia was noted in patients with NSAID-induced small intestinal injury, but albumin levels were normal (Table 1).
Table 1. Clinical Characteristics of Nonsteroidal Anti-Inflammatory Drug-Induced Small Intestinal Injury

\begin{tabular}{lc}
\hline \multicolumn{1}{c}{ Characteristic } & Value \\
\hline Age, yr & $60.6 \pm 14.8$ \\
Male:female & $87(62.1): 53(37.9)$ \\
Type of capsule endoscopy & \\
MiroCam & \\
PilCam SB & \\
Comorbidities & $119(15.0)$ \\
Hypertension & \\
Diabetes & $60(42.9)$ \\
Cardiovascular disease & $26(18.6)$ \\
Cerebrovascular disease & $30(21.4)$ \\
Chronic renal disease & $12(8.6)$ \\
Arthritis & $8(5.7)$ \\
Follow-up period, mo & $27(19.2)$ \\
Initial blood test & $15.9 \pm 19.0(0-106)$ \\
Hemoglobin, g/dL & \\
Albumin, g/dL & $9.6 \pm 2.7$ \\
\hline
\end{tabular}

Data are presented as mean \pm SD (range) or number (\%).

\section{CE findings of NSAID-induced small intestinal injury}

The patients were examined by means of the PilCam $\mathrm{SB}^{\circledR}$ (85.0\%) or MiroCam ${ }^{\circledR}$ (15.0\%). The most common indication for $\mathrm{CE}$ was bleeding of unknown origin. Fifty-six patients were examined for obscure, overt GI bleeding (40\%) and four patients were examined for obscure, occult GI bleeding (2.9\%). The presence of melena $(n=34,24.3 \%)$ was the second most common indication (Table 2). The most common key CE finding was ulceration $(n=82,58.6 \%)$. Erosions and mucosal erythema were also 

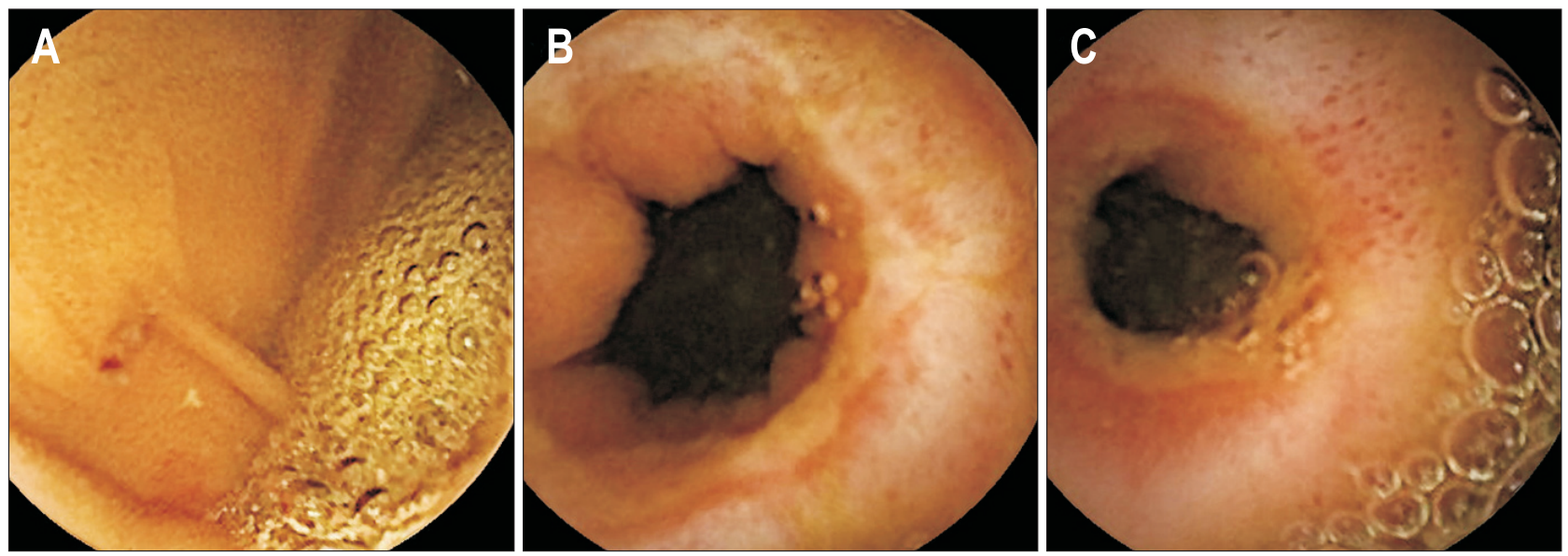

Fig. 3. Capsule endoscopic findings of nonsteroidal anti-inflammatory drug-induced small intestinal injury. Erosions (A), ulcerations (B), and strictures $(\mathrm{C})$ were noted.

Table 3. The Clinical Features of Six Recurrent Cases of Nonsteroidal Anti-Inflammatory Drug-Induced Small Intestinal Injury

\begin{tabular}{|c|c|c|c|c|c|c|c|}
\hline $\begin{array}{c}\text { Case } \\
\text { no. }\end{array}$ & $\begin{array}{c}\text { Sex/ } \\
\text { age, yr }\end{array}$ & $\begin{array}{l}\text { Type of } \\
\text { NSAID }\end{array}$ & $\begin{array}{c}\text { Symptoms } \\
\text { when recurred }\end{array}$ & $\begin{array}{l}\text { Examination } \\
\text { performed } \\
\text { when recurred }\end{array}$ & $\begin{array}{c}\text { Treatment } \\
\text { when recurred }\end{array}$ & $\begin{array}{l}\text { Sort of treatment } \\
\text { medication }\end{array}$ & $\begin{array}{l}\text { Period until } \\
\text { recurrence, mo }\end{array}$ \\
\hline 1 & $\mathrm{M} / 67$ & Aspirin & Melena & EGD, CFS & Add medication & PPI & 22 \\
\hline 2 & $\mathrm{~F} / 62$ & Aspirin+NSAID & Hematochezia & EGD, CFS & Add medication & Rebamipide & 28 \\
\hline 3 & $\mathrm{~F} / 69$ & Aspirin & Melena & None & Add medication & Iron supplement & 14 \\
\hline 4 & $\mathrm{M} / 69$ & Aspirin & Melena & EGD, CFS & Add medication & PPI & 1 \\
\hline 5 & $\mathrm{M} / 74$ & Aspirin & Anemia & None & Add medication & Iron supplement & 27 \\
\hline 6 & $\mathrm{M} / 60$ & Aspirin & Anemia & EGD, CFS, CE & Add medication & Iron supplement & 9 \\
\hline
\end{tabular}

NSAID, nonsteroidal anti-inflammatory drug; M, male; EGD, esophagogastroduodenoscopy; CFS, colonofiberoscopy; PPI, proton pump inhibitor; F, female; CE, capsule endoscopy.

Table 2. Indication of Capsule Endoscopy in Patients with Nonsteroidal Anti-Inflammatory Drug-Induced Small Intestinal Injury

\begin{tabular}{lc}
\hline \multicolumn{1}{c}{ Indication } & No. (\%) \\
\hline Obscure overt GI bleeding & $56(40.0)$ \\
Melena & $34(24.3)$ \\
Hematochezia & $15(10.7)$ \\
Abdominal pain & $14(10.0)$ \\
Anemia & $7(5.0)$ \\
Obscure occult GI bleeding & $4(2.9)$ \\
Etc. (diarrhea, abnormal findings in CT, & $10(7.1)$ \\
small bowel series) & \\
\hline
\end{tabular}

GI, gastrointestinal; CT, computed tomography.

common (Fig. 3). Other lesions found in patients with NSAIDinduced small intestinal injury included luminal narrowing and stenosis, small bowel submucosal tumor, and small bowel polyps (Table 3). The lesions were located frequently in the jejunum ( $\mathrm{n}=74,52.8 \%)$ and ileum $(\mathrm{n}=39,27.9 \%)$.

\section{Treatment for NSAID-induced small intestinal injury}

Most patients stopped NSAID therapy after diagnosis ( $\mathrm{n}=91$, 71.1\%). However, 28 patients (21.9\%) continued to take NSAIDs following their diagnosis. Aspirin was the most common medication used among the patients who continued to take NSAIDs. Following diagnosis, most of the patients were treated by noninvasive methods, such as simply discontinuing NSAID therapy $(\mathrm{n}=13,10.2 \%)$ or were prescribed additional medication $(\mathrm{n}=44$, $34.4 \%$ ), or both ( $\mathrm{n}=52,40.6 \%)$. Only three cases $(2.3 \%)$ underwent endoscopic treatment, and none of the patients had surgery (Fig. 4). A proton pump inhibitor (PPI) (n=61, 63.6\%) was most commonly prescribed additional medication. Rebamipide ( $\mathrm{n}=21,21.9 \%)$, an $\mathrm{H}_{2}$-receptor blocker $(\mathrm{n}=18,18.6 \%)$, and misoprostol $(n=10,10.4 \%)$ were also commonly prescribed therapeutic drugs in patients with NSAID-induced small intestinal injury.

\section{Clinical features of recurrent and nonrecurrent cases of NSAID-induced small intestinal injury}

Only six NSAID-induced small intestinal injury cases recurred during the follow-up period. The mean age of the recurrent 


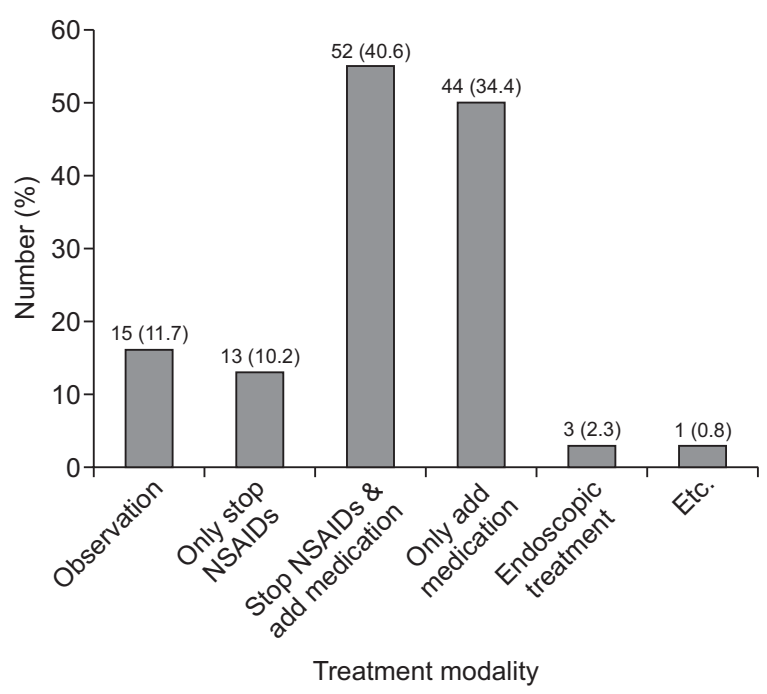

Fig. 4. Treatment modalities of patients with nonsteroidal antiinflammatory drug (NSAID)-induced small intestinal injury. Data are presented as number (\%). cases was $66.8 \pm 5.1$ years (range, 60 to 74 years). Four of the six patients revisited the hospital complaining of symptoms of bleeding, and recurred or continued anemia was found in two cases. Of the recurring patients, only one underwent CE again. The majority of patients were supposed to recur with NSAIDinduced small intestinal injury based on negative findings in studies other than CE. All six recurrent cases continued to take aspirin after initial diagnosis, but, only $21.2 \%$ of patients continued to take aspirin in nonrecurrent cases $(p=0.001)$. Aspirin was the most commonly prescribed medication for the recurrent cases. One patient was taking a NSAID other than aspirin due to severe pain from arthritis. Cardiovascular disease, such as ischemic heart disease, was the reason for taking medication in four of six recurrent cases. All patients recovered by conservative management without endoscopic treatment or surgery (Table 3).

The clinical characteristics of cases involving recurrence and nonrecurrence were observed (Table 4). Mean age was significantly higher in recurrent than in nonrecurrent cases $(66.8 \pm 5.1$ years vs $60.1 \pm 14.8$ years, $p=0.023$ ). No significant difference was observed in the male to female distribution. The initial drug

Table 4. Comparison of Recurrent and Nonrecurrent Patients

\begin{tabular}{|c|c|c|c|}
\hline Variable & Nonrecurrent cases $(n=110)$ & Recurrent cases $(\mathrm{n}=6)$ & p-value \\
\hline Age, yr & $60.1 \pm 14.8$ & $66.8 \pm 5.1$ & 0.023 \\
\hline Male:female & $65: 45$ & $4: 2$ & 0.949 \\
\hline Initial drug history & & & 0.349 \\
\hline Aspirin & $38(34.5)$ & $4(66.7)$ & \\
\hline NSAID (except aspirin) & $42(38.2)$ & $2(33.3)$ & \\
\hline Aspirin+NSAID & $12(10.2)$ & 0 & \\
\hline Continuance of NSAID & & & 0.001 \\
\hline Discontinue & $80(67.8)$ & 0 & \\
\hline Continue & $25(21.2)$ & $6(100.0)$ & \\
\hline \multicolumn{4}{|l|}{ Underlying disease } \\
\hline Hypertension & $47(42.7)$ & $6(100.0)$ & 0.023 \\
\hline Diabetes mellitus & $17(15.5)$ & $3(50.0)$ & 0.092 \\
\hline Cardiovascular disease & $24(21.8)$ & $3(50.0)$ & 0.279 \\
\hline Chronic renal disease & $4(3.6)$ & $1(16.7)$ & 0.304 \\
\hline Arthritis & $24(21.8)$ & $2(33.3)$ & 0.789 \\
\hline Cerebrovascular disease & $9(8.2)$ & 0 & 0.742 \\
\hline Hemoglobin level & $9.62 \pm 2.59$ & $8.35 \pm 2.31$ & 0.244 \\
\hline Key findings of capsule endoscopy & & & 0.001 \\
\hline Ulcer & $69(62.7)$ & $2(33.3)$ & \\
\hline Erosion & $25(22.7)$ & $1(16.7)$ & \\
\hline Mucosal erythema & $7(6.4)$ & 0 & \\
\hline Mucosal spotty hemorrhage & $3(2.7)$ & $1(16.7)$ & \\
\hline Diffuse small bowel bleeding & 0 & $1(16.7)$ & \\
\hline Angiodysplasia & 0 & $1(16.7)$ & \\
\hline
\end{tabular}

Data are presented as mean \pm SD or number $(\%)$.

NSAID, nonsteroidal anti-inflammatory drug. 
history was not different between the two groups. However, taking aspirin was more common among recurrent cases. But, the difference was not statistically significant. Of the recurrent cases, one patient who was initially diagnosed with NSAID-induced small intestinal injury due to taking an NSAID because of arthritis changed medication to aspirin after suffering ischemic heart disease. Including this case, the majority of recurrent patients were taking aspirin. Hypertension was more common in recurrent cases (42.7\% vs 100\%, $\mathrm{p}=0.023$ ), but other underlying diseases were not different. As the key CE findings were significantly different between recurrent and nonrecurrent cases, ulcerations and erosions were more common in nonrecurrent than recurrent cases. Ulcerations were commonly noted in recurrent cases; however, other findings, such as diffuse small bowel bleeding and mucosal spotty hemorrhage, were also noted.

\section{Change in diagnosis}

Twelve patients who were initially diagnosed with NSAIDinduced small intestinal injury had their changed diagnosis after other diseases were identified during the follow-up period. Among these 12 cases, five were due to initial misdiagnosis. Chiefly, errors in drug history of the patients resulted in the misdiagnosis. The adjusted diagnoses were as follows: angiodysplasia, ischemic enteritis, tuberculosis enteritis, Dieulafoy's lesion, gastrointestinal stromal tumor, idiopathic ulceration, Behçet's disease, and ileal erosion.

\section{DISCUSSION}

NSAIDs are one of the most commonly prescribed drugs due to their analgesic and anti-inflammatory properties. They are used to reduce fever and treat pain or inflammation caused by chronic conditions such as rheumatoid arthritis and osteoarthritis. ${ }^{13}$ Previous studies have focused primarily on their upper GI events. NSAID-induced small intestinal injury has gained more attention due to the introduction of new diagnostic modalities, such as CE and double-balloon endoscopy. ${ }^{9}$ However, little is known about the mechanism of NSAID-induced small intestinal injury. Our study focused not only on the prevalence and characteristics of patients diagnosed with NSAID-induced small intestinal injury but included data accumulated during long-term observations of these patients, such as recurrence and changes in diagnoses.

The number of patients undergoing CE for various reasons is increasing. However, CE remains a relatively rarely performed examination. Thus, the Korean Gut Image Study Group founded the capsule endoscopy nationwide database registry in 2002 to accumulate and analyze clinical data of CE. The Study Group for Double-Balloon Endoscopy reported that NSAID-induced small intestinal injury occurred in 51\% of the patients taking NSAIDs, based on the registry data accumulated during a 2-year period beginning in 2008. ${ }^{14}$ However, this study did not include long-term follow-up data. Our study is the first report to analyze the data collected from the nationwide database registry of CE and include long-term follow-up data.

Only six cases (4.3\%) in the capsule endoscopy nationwide database registry in Korea recurred as NSAID-induced small intestinal injury during the follow-up period from 2002 to 2012. Most of these patients recovered without endoscopic or surgical intervention. Patients were treated with medications even after recurrence. Initial drug history, initial hemoglobin level, and the treatment options were not different between recurrent and nonrecurrent cases. However, older age and hypertension were positive predictive factors for recurrence. More atypical CE findings, such as diffuse small bowel bleeding, mucosal spotty hemorrhage, and angiodysplasia were observed in the recurrent group. Thus, older patients, those with hypertension, and atypical CE findings were monitored carefully because of the high probability of recurrence regardless of drug history or treatment options. Continuous use of aspirin was the most significant factor to cause recurrence. But recurrence of NSAID bleeding is not frequent and most of recurred bleeding can be managed conservatively similar to first episode of bleeding.

Among the 140 patients diagnosed with NSAID-induced small intestinal injury, 12 were diagnosed with another disease during the follow-up period. Five of these cases were misdiagnosed initially. Mistakes in taking the drug history were the major cause of misdiagnosis. Seven cases initially diagnosed as NSAID-induced small intestinal injury were changed to other diseases. After its diagnosis, NSAID-induced small intestinal injury reacted well to medication and conservative treatment, and the recurrence rate was relatively low. Initial history taking was important because the diagnosis of NSAID-induced small intestinal injury is largely dependent on drug history.

It is important to evaluate the long-term efficacy of the drugs prescribed for patients with NSAID-induced small intestinal injury. Administration of NSAIDs is known to induce upper GI complications, and PPIs prevent their occurrence. ${ }^{15,16}$ However, to date, no medications that could prevent or heal NSAID-induced small intestinal injuries were available. PPIs are the most effective medications for gastroduodenal bleeding and ulcers but cannot be expected to be clinically efficacious for small intestinal mucosal injury, as acid is not secreted into the small intestine. ${ }^{17}$ PPIs worsen NSAID-induced small intestinal injury in animals, and emerging evidence indicates that such worsening may occur in some humans. ${ }^{18,19}$ Managing patients with NSAIDinduced small intestinal injury relies empirically on misoprostol and rebamipide. Misoprostol has demonstrated positive therapeutic effects in NSAID-induced small intestinal injury. ${ }^{20}$ Niwa et al. ${ }^{17}$ found that subjects who received an NSAID and placebo demonstrated a significantly greater number of small intestine mucosal injuries than those who received rebamipide. However, these studies were relatively small, and therapeutic efficacy was evaluated over the short-term. In the present study, the PPI, re- 
bamipide, $\mathrm{H}_{2}$ blocker, and misoprostol were the most commonly prescribed drugs in patients with NSAID-induced small intestinal injury. When the influence of the drugs on disease recurrence was evaluated, no significant differences were observed.

To our knowledge, this is the first study about the long-term outcome of NSAID-induced small intestinal injury assessed by CE based on a nationwide multicenter data registry.

Several limitations of this study should be discussed. First, it was a retrospective analysis. We collected data from the capsule endoscopy nationwide database registry. Furthermore, we reviewed the charts of patients and conducted telephone interviews to collect data. Hence, recall error could have influenced the results. Second, we did not assess the severity of NSAID-induced small intestinal injury in each patient. Recurrence may be dependent on disease severity at original presentation. However, we are uncertain whether the recurrence rate was dependent on the severity of NSAID-induced small intestinal injury. Third, a complete follow-up of all patients was not possible. Therefore, 116 of 140 patients were enrolled in this analysis of recurrence. And all cases with anemia or occult obscure gastrointestinal bleeding did not undergo follow-up complete blood count regularly after the first presentation. So, we think there were more current cases that did not be revealed.

In conclusion, the recurrence of NSAID-induced small intestinal injury was infrequent regardless of conservative management, such as discontinuing NSAID therapy or administration of additional medications. Hence, an initially accurate diagnosis by performing CE and a thorough medication history were important. Although changes in diagnosis following an initially accurate diagnosis were infrequent; however, some patients were subsequently diagnosed with another disease during the followup period. Therefore, differential diagnoses should be considered carefully when evaluating NSAID-induced small intestinal injury.

\section{CONFLICTS OF INTEREST}

No potential conflict of interest relevant to this article was reported.

\section{REFERENCES}

1. Laine L. Approaches to nonsteroidal anti-inflammatory drug use in the high-risk patient. Gastroenterology 2001;120:594-606.

2. Bjarnason I, Peters TJ. Intestinal permeability, non-steroidal antiinflammatory drug enteropathy and inflammatory bowel disease: an overview. Gut 1989;30:22-28.

3. Hawkey CJ. Non-steroidal anti-inflammatory drugs and peptic ulcers. BMJ 1990;300:278-284.

4. Kameda N, Higuchi K, Shiba M, et al. A prospective, single-blind trial comparing wireless capsule endoscopy and double-balloon enteroscopy in patients with obscure gastrointestinal bleeding. J
Gastroenterol 2008;43:434-440.

5. Yamamoto H, Sekine Y, Sato Y, et al. Total enteroscopy with a nonsurgical steerable double-balloon method. Gastrointest Endosc 2001;53:216-220.

6. Sunada K, Yamamoto H. Double-balloon endoscopy: past, present, and future. J Gastroenterol 2009;44:1-12.

7. Iddan G, Meron G, Glukhovsky A, Swain P. Wireless capsule endoscopy. Nature 2000;405:417.

8. Rami E. Capsule endoscopy: where are we at 2011 and where are we headed? Intest Res 2012;10:235-243.

9. Lim YJ, Yang CH. Non-steroidal anti-inflammatory drug-induced enteropathy. Clin Endosc 2012;45:138-144.

10. Goldstein JL, Eisen GM, Lewis B, et al. Video capsule endoscopy to prospectively assess small bowel injury with celecoxib, naproxen plus omeprazole, and placebo. Clin Gastroenterol Hepatol 2005;3:133-141.

11. Graham DY, Opekun AR, Willingham FF, Qureshi WA. Visible small-intestinal mucosal injury in chronic NSAID users. Clin Gastroenterol Hepatol 2005;3:55-59.

12. Kurokawa S, Katsuki S, Fujita T, et al. A randomized, doubleblinded, placebo-controlled, multicenter trial, healing effect of rebamipide in patients with low-dose aspirin and/or non-steroidal anti-inflammatory drug induced small bowel injury. J Gastroenterol 2014;49:239-244.

13. Watanabe T, Tanigawa T, Nadatani Y, et al. Risk factors for severe nonsteroidal anti-inflammatory drug-induced small intestinal damage. Dig Liver Dis 2013;45:390-395.

14. Matsumoto T, Kudo T, Esaki M, et al. Prevalence of non-steroidal anti-inflammatory drug-induced enteropathy determined by double-balloon endoscopy: a Japanese multicenter study. Scand J Gastroenterol 2008;43:490-496.

15. Lanas A, García-Rodriguez LA, Arroyo MT, et al. Effect of antisecretory drugs and nitrates on the risk of ulcer bleeding associated with nonsteroidal anti-inflammatory drugs, antiplatelet agents, and anticoagulants. Am J Gastroenterol 2007;102:507-515.

16. Lanza FL, Chan FK, Quigley EM; Practice Parameters Committee of the American College of Gastroenterology. Guidelines for prevention of NSAID-related ulcer complications. Am J Gastroenterol 2009;104:728-738.

17. Niwa Y, Nakamura M, Ohmiya N, et al. Efficacy of rebamipide for diclofenac-induced small-intestinal mucosal injuries in healthy subjects: a prospective, randomized, double-blinded, placebocontrolled, cross-over study. J Gastroenterol 2008;43:270-276.

18. Wallace JL. Mechanisms, prevention and clinical implications of nonsteroidal anti-inflammatory drug-enteropathy. World J Gastroenterol 2013;19:1861-1876.

19. Wallace JL, Syer S, Denou E, et al. Proton pump inhibitors exacerbate NSAID-induced small intestinal injury by inducing dysbiosis. Gastroenterology 2011;141:1314-1322.

20. Watanabe T, Sugimori S, Kameda N, et al. Small bowel injury by low-dose enteric-coated aspirin and treatment with misoprostol: a pilot study. Clin Gastroenterol Hepatol 2008;6:1279-1282. 